Pesq. Vet. Bras. 37(7):729-733, julho 2017

DOI: $10.1590 / \mathrm{S} 0100-736 \mathrm{X} 2017000700013$

\title{
Eficácia da ciclosporina no controle da dermatite atópica em cães ${ }^{1}$
}

\author{
Adriano Souza Neto ${ }^{2 *}$, Marconi R. de Farias ${ }^{3}$, Claudia T. Pimpão ${ }^{4}$, Juliany G. Quitzan ${ }^{5}$ \\ e Amanda Anater ${ }^{6}$
}

\begin{abstract}
Souza Neto A., Farias M.R., Pimpão C.T., Quitzan J.G. \& Anater A. 2017. [Efficacy of cyclosporine in the control of atopic dermatitis in dogs.] Eficácia da ciclosporina no controle da dermatite atópica em cães. Pesquisa Veterinária Brasileira 37(7):729-733. Programa de Pós-Graduação em Ciência Animal, Escola de Ciências Agrárias e Ambientais, Pontifícia Universidade Católica do Paraná, BR-376 Km 14, São José dos Pinhais, PR 83010500, Brazil. E-mail: adriano.asn@hotmail.com

Atopic dermatitis is an itchy, chronic inflammatory skin disease of genetic origin, resulting from loss of the physical barrier function of the skin and hyper-reactivity to environmental allergens, trofoallergens, microbial allergens and to primary irritants. The efficacy of cyclosporine in the control of pruritus and lesions associated with atopic dermatitis in dogs was evaluated. Twenty-four dogs with atopic dermatitis were selected, based on Favrot et al.'s criteria (2010). They were divided into two groups of 12 dogs, where Group 1 received cyclosporine $(5 \mathrm{mg} / \mathrm{kg} / \mathrm{vo} / 24 \mathrm{~h})$, and Group 2 was treated with prednisone $(0.5 \mathrm{mg} / \mathrm{kg} /$ vo/24h) in decreasing doses, both for 60 days. The animals were continuously evaluated, and theirits lesional symptomatology scores were based on a Cadesi-03 scale, set on days 0,30 and 60. Pruritus scores of each dog, based on Rybnicek's criteria, were weekly evaluated, from day 0 to day 63 . All collected data were analyzed by the nonparametric Kruskal-Wallis' test, followed by Dunn's test, and for the analysis between the groups, considered the minimum significance level of 5\%, t-test was used. Cyclosporin had similar efficacy in lesional control in the group which received prednisone on day $(+30)(\mathrm{p}<0.05)$ and on the day $(+60)(p<0.001)$ of treatment, compared with day zero. A significant difference of the itching score was observed on days $+28,+35,+42,+49,+56$ and $+63(\mathrm{p}<0.001)$, and on day $+21(\mathrm{p}<0.01)$ when compared to initial treatment. However, its efficacy was lower than Group 2, from 42 days of evaluation on, keeping such difference on days $+49,+56$ and +63 $(p>0.05)$. Although cyclosporin have been less effective in controlling itching, it remained at acceptable levels, and its continued use was not associated with significant side effects.
\end{abstract}

INDEX TERMS: Cyclosporine, dermatitis, dogs, CADESI, pruritus, Rybnicek, genodermatosis, corticoid.

RESUMO.- A dermatite atópica é uma dermatopatia inflamatória, pruriginosa, crônica, de origem genética, resultante da perda da função de barreira física da pele e da hiperreatividade à alérgenos ambientais, trofoalérgenos,

\footnotetext{
${ }^{1}$ Recebido em 17 de julho de 2015.

Aceito para publicação em 9 de novembro de 2016.

${ }^{2}$ Discente do Programa de Pós-Graduação em Ciência Animal, área de concentração Saúde, Tecnologia e Produção Animal, Escola de Ciências Agrárias e Ambientais, Pontifícia Universidade Católica do Paraná (PUC), BR-376 Km 14, São José dos Pinhais, PR 83010-500, Brasil. *Autor para correspondência: adriano.asn@hotmail.com

${ }^{3}$ Docente do Departamento de Clinica Médica Veterinária, Escola de Ciências Agrárias e Ambientais, PUC, BR-376 Km 14, São José dos Pinhais, PR 83010-500, Brasil. E-mail: marconi.puc@terra.com.br
}

alérgenos microbianos e a irritantes primários. Este estudo avaliou a eficácia da ciclosporina no controle do prurido e das lesões associadas à dermatite atópica em cães. Selecionaram-se 24 cães com diagnóstico de dermatite atópica ba-

\footnotetext{
${ }^{4}$ Docente do Departmento de Farmacologia e Toxicologia Veterinária, Escola de Ciências Agrárias e Ambientais, Pontifícia Universidade Católica do Paraná (PUC), BR-376 Km 14, São José dos Pinhais, PR 83010500, Brasil.

${ }^{5}$ Docente do Departyamento de Clinica Médica e Cirurgia Veterinária, Universidade Estadual Paulista Júlio de Mesquita Filho (Unesp), Cx. Postal 237, Botucatu, SP 18618-000, Brasil.

${ }^{6}$ Discente de Graduação em Medicina Veterinária e Bolsista de Iniciação Científica (PIBITI), PUC, BR-376 Km 14, São José dos Pinhais, PR 83010500, Brasil.
} 
seados nos critérios de Favrot et al. (2010), os quais foram divididos em dois grupos de 12 cães, onde o Grupo 1, recebeu ciclosporina ( $5 \mathrm{mg} / \mathrm{kg} / \mathrm{vo} / 24 \mathrm{~h}$ ), e o Grupo 2 , foi tratado com prednisona $(0,5 \mathrm{mg} / \mathrm{kg} / \mathrm{vo} / 24 \mathrm{~h})$ em doses decrescentes, ambos por 60 dias. Os animais foram continuamente avaliados, e seus escores sintomato-lesionais, baseados na escala de CADESI-03, estabelecidos nos dias 0, 30 e 60 . Em adição, os escores de prurido de cada animal, baseado nos critérios de Rybnicek, foram semanalmente avaliados, do dia 0 ao 63. Todos os dados coletados foram analisados pelo teste não paramétrico de Kruskal-Wallis, seguido do teste de Dunn's e para as análises entre os grupos foi utilizado o teste $\mathrm{t}$, considerado o nível de significância mínimo de $5 \%$. A ciclosporina teve uma eficácia similar, no controle lesional, ao grupo que recebeu prednisona no dia $(+30)$ $(\mathrm{p}<0,05)$ e no dia $(+60)(\mathrm{p}<0,001)$ do tratamento, em relação ao dia zero. Uma diferença significativa do escore do prurido foi observada nos dias $+28,+35,+42,+49,+56$ e $+63(\mathrm{p}<0,001)$, e no dia $+21(\mathrm{p}<0,01)$ em relação ao momento inicial do tratamento, porém sua eficácia foi inferior ao Grupo 2, a partir do $42^{\circ}$ dia de avaliação, mantendo-se esta diferença nos dias $+49,+56$ e $+63(p>0,05)$. Apesar da ciclosporina ter sido menos eficaz no controle do prurido, este se manteve em níveis aceitáveis, e seu uso contínuo não foi associado a efeitos colaterais relevantes.

TERMOS DE INDEXAÇÃO: Ciclosporina, dermatite atópica, cães, CADESI, prurido, Rybnicek, genodermatose, corticóide.

\section{INTRODUÇÃO}

A dermatite atópica é uma doença de pele inflamatória, crônica, recorrente e pruriginosa (Deboer 2004, Cork et al. 2006), e embora de etiologia multifatorial, sua fisiopatologia está relacionada à mutações genéticas que conduzem a distúrbios da função de barreira tegumentar, a defeitos na resposta imune antimicrobiana e a hiper-reatividade cutânea a alérgenos ambientais, antígenos microbianos, irritantes e trofoalérgenos (Homey et al. 2006, Nuttal 2008, Olivry et al. 2010).

O principal sinal clínico da dermatite atópica é o prurido, que possui caráter moderado a grave, crônico, perene, primário ou pode estar associado a erupções papulares, máculas ou manchas eritematosas (Hillier 2002, Olivry et al. 2005, Akdis et al. 2006). As principais áreas da pele envolvidas são a face (especialmente as regiões perioculares, perilabiais, mentonianas e o plano nasolabial); o epitélio dos condutos auditivos e a superfície côncava dos pavilhões auriculares; a superfície ventral (região cervical ventral, axila, abdome e virilha); a porção distal dos membros (superfícies dorso-digitais e interdigitais dorsal e ventral), as áreas flexurais (flexuras carpianas, tíbio-társicas e anticubitais) e a região perineal (Favrot 2009).

0 tratamento em geral é contínuo e multifatorial, baseado na associação de terapia tópica e sistêmica que minimizem os sinais clínicos e não seja associada a efeitos colaterias (Deboer 2008).

Os glicocorticóides sistêmicos, apesar de muito eficazes, possuem uma pletora de efeitos colaterais e é comum a recidiva dos sinais clínicos, quando estes são descontinua- dos, o que induz ao seu uso contínuo e abusivo pelo médico veterinário ou pelos proprietários (Del Rosso \& Friedlander 2005, Akdis et al. 2006).

A ciclosporina é um polipeptídio cíclico, lipossolúvel, derivado do fungo Tolypocladium inflatum gams (Steffan et al. 2004). Este fármaco liga-se a uma proteína intracelular, a ciclofilina-1, e inibe a calcineurina, enzima que codifica a interleucina 2. A ausência desta interleucina impede a ativação e a proliferação de linfócitos T, frente a outras citocinas, como interleucina 4 e ao interferon gamma (INF- $\gamma$ ) (Guaguère et al. 2004).

A eficácia e segurança da ciclosporina no controle da DAC foi documentada por diversos autores (Fontaine \& Olivry 2001, Olivry et al. 2002a, 2002b, Olivry \& Mueller 2003, Burton et al. 2004, Radowicz \& Power 2005, Steffan et al. 2006, Lucas et al. 2007, Yazbek \& Larsson 2012), e com taxas de 71 a $75 \%$ de satisfação do proprietário (Yazbek \& Larsson 2012).

0 presente estudo teve como objetivo avaliar a eficácia de uma fórmula micro-emulsificada de ciclosporina, na involução e controle do quadro sintomato-lesional e do prurido associado à dermatite atópica em cães.

\section{MATERIAL E MÉTODOS}

Foi realizado um estudo longitudinal, com amostras clínicas de conveniência e aleatorizado para a avaliação da eficácia da ciclosporina de uso veterinário, comparada com a prednisona em cães com diagnóstico de dermatite atópica, atendidos na Unidade Hospitalar para Animais de Companhia da Pontifícia Universidade Católica do Paraná (PUCPR).

Este estudo foi aprovado pela Comissão de Ética no Uso de

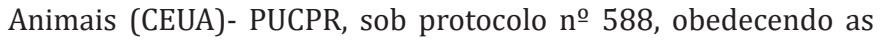
normas de boas práticas clínicas com a utilização de animais em protocolos experimentais.

Os cães incluídos no estudo apresentavam prurido crônico, primário, perene, de intenso a grave, e obedecia, seis ou mais dos critérios estabelecidos por Favrot et al. (2010) para o diagnóstico de dermatite atópica, como:

Aparecimento dos sinais antes de três anos de idade;

Cães domiciliados a maior parte do tempo;

Histórico de prurido crônico, perene, primário (sini material) e responsivo a corticóides;

Lesões nas porções distais de membro torácico;

Lesões em pavilhões auriculares;

Ausência de lesões nas bordas dos pavilhões auriculares;

Ausência de lesões em região lombo-sacral.

Estes cães pertenciam a ambos os gêneros e foram incluídos, independentemente da raça. Em adição, o prurido destes animais não apresentava um controle adequado dos sinais clínicos, após adequada exclusão de ectoparasitas, ao controle da piodermite e malasseziose eczematizantes e a exclusão dietética, por seis a oito semanas, com proteína original.

O protocolo experimental foi dividido em dois grupos:

Grupo CP. Composto por doze cães com dermatite atópica, que receberam ciclosporina (Atopex ${ }^{\circledR} 25$, Ciclosporina 25mg, Cipla, Indía. Atopex ${ }^{\circledR}$ 50, Ciclosporina 50mg, Cipla, Indía), na dose de 5mg/ $\mathrm{kg}$, a cada 24 horas, por via oral, durante 60 dias, sempre realizada com o animal em jejum de no mínimo duas horas. Juntamente, foi administrado prednisona $0,5 \mathrm{mg} / \mathrm{kg}$ a cada 24 horas, por via oral, durante sete dias e, logo após, houve redução de sua dosagem para $0,5 \mathrm{mg} / \mathrm{kg}$ a cada 48 horas, por mais sete dias. A administração de ciclosporina foi mantida como monoterapia pelos 46 dias adicionais. 
Grupo 2. Este grupo foi composto por doze cães com dermatite atópica, que receberam Prednisona (Prednisona 5mg, Neo Química, Brasil. Prednisona 20mg, Neo Química, Brasil) na dose de $0,5 \mathrm{mg} / \mathrm{kg}$, a cada 24 horas, por via oral durante sete dias. Após foi administrado Prednisona 0,5mg/kg a cada 48 horas por 14 dias e em seguida se manteve um protocolo de manutenção, onde a Prednisona foi administrada na dose de $0,5 \mathrm{mg} / \mathrm{kg}$ a cada 72 horas durante 39 dias.

Cada cão incluído no estudo foi submetido ao exame clínico dermatológico pelo pesquisador, com completo delineamento sintomato-lesional e estabelecimento de escores pelos critérios de CADESI-03 (Olivry et al. 2007), nos dias 0, +30 e +60, durante a administração da ciclosporina e da prednisona.

0 escore de prurido foi avaliado semanalmente pelo pesquisador, obedecendo aos critérios de Rybnicek (2009), entre os dias 0 e 63 do estudo.

Os dados iniciais (dia 0) bem como os dados obtidos durante os 60 dias de tratamento foram então comparados e analisados, sendo cada animal controle dele mesmo na avaliação da eficácia.

Os dados obtidos foram analisados pelo teste não paramétrico de Kruskal-Wallis, seguido de teste de Dunn's, adotando-se o nível de significância de $5 \%(\alpha=0,05)$. Para as análises entre os grupos foi utilizado o teste t. Todas as análises estatísticas foram realizadas com a utilização do software Graphpad Prism versão 3.0.

\section{RESULTADOS}

A ciclosporina foi bem tolerada pelos animais, e não houve dificuldade na sua administração. Os efeitos colaterais observados em $2(12)(16,67 \%)$ dos pacientes foram episódios esporádicos de vômito durante dois dias, os quais involuíram espontaneamente, sem a necesidade de interrupção da administração do fármaco.

Em relação à eficácia, a ciclosporina no presente estudo, de acordo com a escala de CADESI, foi responsável por uma involução sintomato-lesional no dia $(+30)$ e no dia $(+60)$ $(p<0,001)$ do tratamento, em relação ao dia zero (Fig.1).

A involução das lesões ocorreu de maneira mais acentuada nos primeiros 14 dias, quando seu uso foi associado ao de corticosteróides.

Durante o tratamento dos cães com ciclosporina foi observada uma tendência de redução do escore de prurido, segundo os critérios de Rybnicek (2009), principalmente a partir do $14^{\circ}$ dia e ao longo dos 63 dias de estudo, com

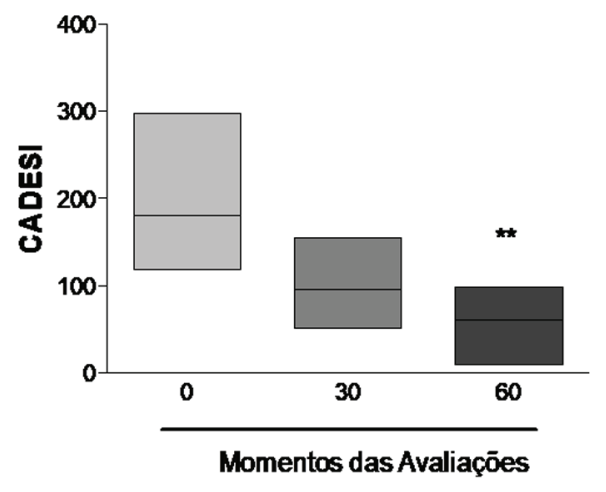

** $p<0,001$ em relaçāo a avaliação no dia 0 . As linhas dentro dos blocos significam as medianas.

Fig.1. Valores das medianas na análise por CADESI dos cães do grupo tratado com ciclosporina nos dias $0,+30$ e +60 .

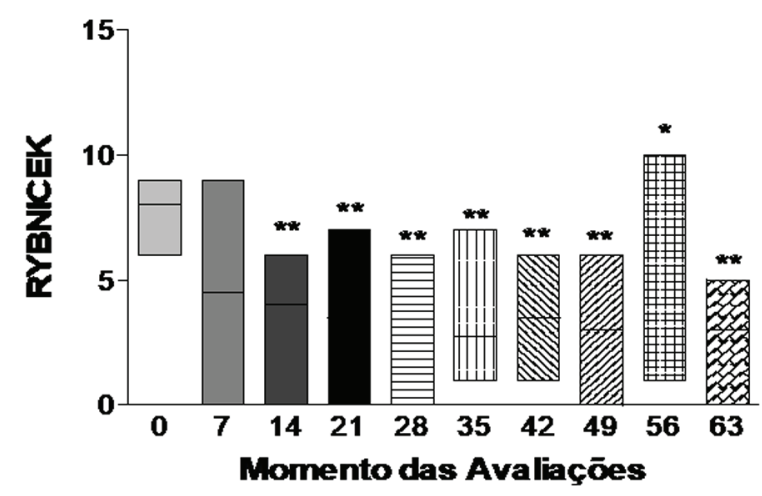

* $p<0,05$ em relação ao momento 0 . ** $p<0,01$ em relação ao momento 0 . As linhas dentro dos blocos representam as medianas.

Fig.2. Valores das medianas na análise por Rybnicek dos cães do grupo tratado com ciclosporina nos dias $0,+7,+14,+21,+28$, $+35,+42,+49,+56$ e +63 .

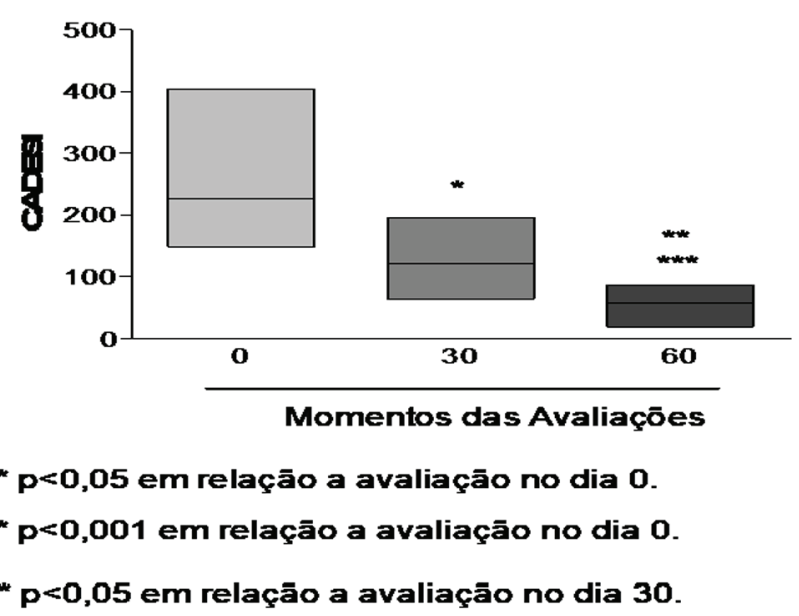

As linhas dentro dos blocos significam as medianas.

Fig.3. Valores das medianas na análise por CADESI dos cães do grupo controle (prednisona) nos dias $0,+30 \mathrm{e}+60$.

uma redução significante $(\mathrm{p}<0,01)$ nos dias $+14,+21,+28$, $+35,+42,+49$ e +63 , e no dia $+56(\mathrm{p}<0,05)$ no momento da avaliação (Fig.2).

A involução do prurido foi associada com a melhora sintomato-lesional dos pacientes, registrada pelo índice de CADESI (Olivry et al. 2007).

Após o início da administração da prednisona, polifagia foi observada em três dos 12 animais (36\%), e poliúria e polidipsia foram observadas em todos os cães.

Em relação à eficácia pela análise de CADESI no grupo controle, houve melhora sintomato-lesional no dia $(+30)$ $(\mathrm{p}<0,05)$ e no dia $(+60)(\mathrm{p}<0,001)$ do tratamento em relação ao dia zero, e na comparação do dia $(+60)(p<0,05)$ em relação ao dia (+30) (Fig.3).

Os valores das medianas no escore de prurido pelos critérios de Rybnicek (2009) dos cães tratados com prednisona foi reduzida a partir do início do tratamento e durante os 63 dias de estudo. Uma diferença significativa do escore do prurido foi observada nos dias $+28,+35,+42,+49,+56$ 


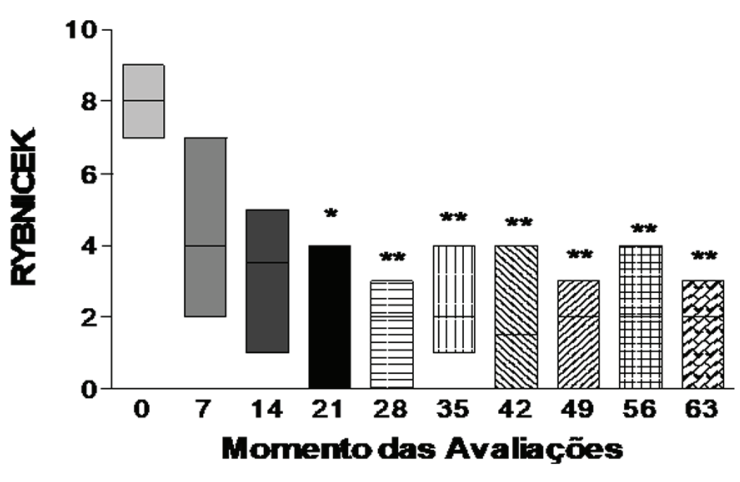

** $p<0,001$ em relação ao momento 0; * $p<0,01$ em relação ao momento 0 . As linhas dentro dos blocos representam as medianas.

Fig.4. Valores das medianas na análise por RYBNICEK dos cães do grupo controle (prednisona) nos dias $0,+7,+14,+21,+28$, $+35,+42,+49,+56$ e +63 .

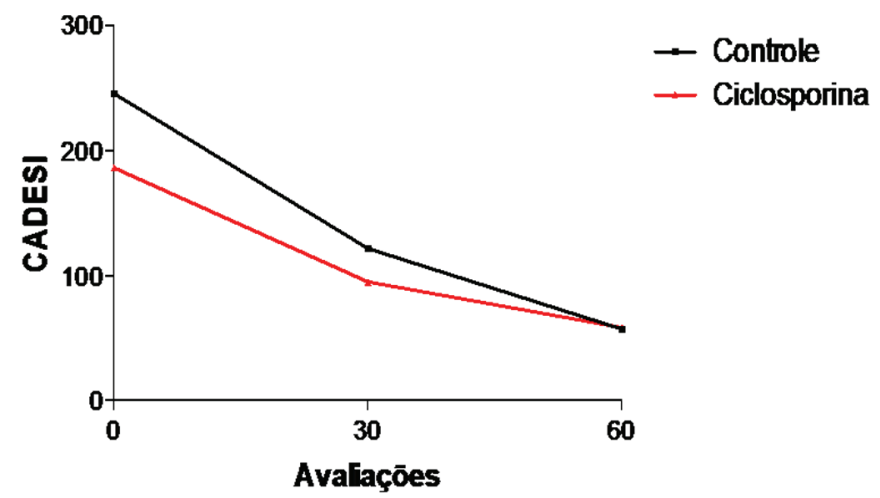

Fig.5. Comparação das medianas na análise por CADESI nos cães do grupo controle (prednisona) e do tratado com ciclosporina nos dias $0,+30$ e +60 .

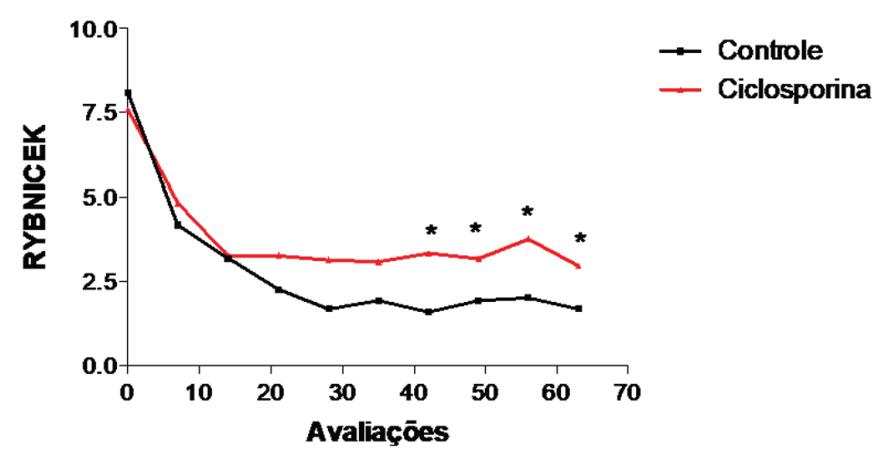

\section{* $p<0,05$ entre os grupos.}

Fig.6. Comparação das medianas na análise por RYBNICEK dos cães do grupo tratado com prednisona, e do grupo com ciclosporina nos dias $0,+7,+14,+21,+28,+35,+42,+49,+56$ e +63 .

e +63 $(p<0,001)$, e no dia $+21(p<0,01)$ em relação ao momento inicial do tratamento (Fig.4).

Quando se comparou os valores das medianas de CADESI entre os dois grupos P (predinisona) e CP (ciclosporina) em relação à involução sintomato-lesional, nenhuma dife- rença estatística foi observada, nos momentos de observação (Fig.5).

Quando se comparou o escore de prurido segundo os critérios de Rybnicek (2009) nos dois grupos experimentais (Fig.6), foram observadas melhores índices de controle da intensidade do prurido no grupo tratado com prednisona, em relação ao grupo com ciclosporina a partir do $42^{\circ}$ dia de avaliação $(\mathrm{p}<0,05)$, mantendo-se esta diferença nos dias $+49,+56$ e +63 .

\section{DISCUSSÃO}

As reações adversas (gastroentéricas) observadas no estudo, associadas à administração de ciclosporina foram baixas, e prevalecem em relação a outros sintomas, porém, geralmente, são comuns, discretas, transitórias e não exigem tratamento ou interrupção precoce da terapia, corroborando com outros estudos (Fontaine \& Olivry 2001, Olivry et al. 2002a, 2002b, Olivry \& Mueller 2003, Burton et al. 2004, Radowicz \& Power 2005, Steffan et al. 2006, Lucas et al. 2007, Yazbek \& Larsson 2012).

A causa deste efeito colateral não está elucidada. Sugere-se que devido ao perfil de dispersão das moléculas do fármaco no intestino delgado (diâmetro médio e distribuição do tamanho das partículas), a disponibilidade de ciclosporina (pico de concentração) depende principalmente da atividade da proteína transportadora intestinal p-glicoproteína (P-gp) e do metabolismo das isoenzimas CYP3A4 e CYP3A5. A expressão de CYP3A, P-gp, e CYP3A está sujeita a polimorfismo genético, que pode afetar as necessidades individuais de dosagem, sua absorção e favorecer à irritabilidade da mucosa e ocorrência de efeitos gastroentéricos, principalmente vômitos (Guaguère et al. 2004).

Outro fator importante preconizado neste estudo, e que pode estar relacionado principalmente às crises eméticas, foi à administração da ciclosporina em jejum em ambos os grupos (C5 e C10), já que em cães, a absorção é ligeiramente retardada quando o medicamento é administrado com alimentos, porém têm-se o risco de o jejum favorecer a irritabilidade da mucosa (Guaguère et al. 2004).

A combinação de ciclosporina com baixas doses de corticosteróides orais foi utilizada no estudo até a indução de uma concentração sérica de ciclosporina capaz de sustentar seus efeitos imunomoduladores. Segundo Hillier (2002) e Guaguère et al. (2004), isto se faz necessário devido ao fato de os efeitos satisfatórios da ciclosporina só serem observados em cães, em média a partir de 21 a 30 dias de uso contínuo. Outro fator relevante para sua associação com os corticosteróides é que sua metabolização é realizada pelo citocromo P-450 e por outros conjuntos enzimáticos oxidativos. Uma vez que os corticóides inibem a ação do citocromo P-450 hepático, este diminui a depuração de ciclosporina, e aumenta seus niveis séricos (Guaguère et al. 2004).

No presente ensaio, mesmo com a retirada dos corticóides, a ciclosporina se manteve eficaz no controle sintomato-lesional a partir do $14^{\circ}$ até o $60^{\circ}$ dia de tratamento, coincidindo com os resultados obtidos por Fontaine \& Olivry (2001), Olivry et al. (2002a, 2002b), Burton et al. (2004), Steffan et al. (2005), Steffan et al. (2006), Lucas et al. (2007), Yazbek \& Larsson (2012). 
Uma das atribuições sugeridas de sua eficácia, no presente estudo, pode ser explicada pela biodisponibilidade e absorção da ciclosporina testada, o qual se apresenta na forma de cápsulas gelatinosas, com a formulação de ciclosporina microemulsificada (ME). De acordo com Guaguère et al. (2004) no homem, a biodisponibilidade da formulação em óleo vegetal é de 20-30\%, já a microemulsificada, aumenta para 30-40\%. Em cães, a formulação (ME) oferece uma biodisponibilidade de $35 \%$, em comparação com 20 $25 \%$ da formulação de óleo vegetal.

A involução do prurido foi associada com a melhora sintomato-lesional dos pacientes, registrada pelo índice de CADESI no estudo. Segundo Guaguère et al. (2004), a redução do prurido e da inflamação na pele, se deve principalmente à ação da ciclosporina sobre a calcineurina, que atua como um fator de transcrição de interleucinas inflamatórias, tais como IL-2, IL-3, IL-4 e TNF-alfa. Assim, a ciclosporina inibe a proliferação de células de Langerhans, diminui a sobrevida e a desgranulação de mastócitos, induz a apoptose de eosinófilos e inibe a liberação de IL-4, IL-5, TNF alfa, IL-3 e IL18, o que conduz a vários efeitos imunomoduladores benéficos e melhora dos sinais clínicos e do prurido associados à dermatite atópica, sem comprometer a função imune do organismo (Guaguère et al. 2004).

Com a ciclosporina, entretanto, o índice de redução de prurido foi considerado adequado pelos proprietários e pela avaliação médica dos pacientes. Em contrapartida, a terapia com corticóide é comumente associada com efeitos colaterais a curto e em longo prazo, e seus índices de redução de prurido não se sustentam com seu uso contínuo, devido à possibilidade de taquifilaxia orgânica, o que induz ao seu uso contínuo e abusivo pelo médico veterinário ou pelos proprietários e reduz a expectativa de vida dos cães (Hillier 2002, Guaguère et al. 2004, Del Rosso \& Friedlander 2005), fato não observado com o uso de ciclosporina.

\section{CONCLUSÃO}

De acordo com os dados obtidos no estudo, podemos concluir que a ciclosporina emulsificada de uso veterinário é eficaz na involução sintomato-lesional e no controle do prurido em cães com dermatite atópica, e não foi associada a efeitos colaterais significativos durante o período de estudo em cães.

\section{REFERÊNCIAS}

Akdis C.A., Akdis M., Bieber T., Bindslev-Jensen C., Boguniewicz M., Eigenmann P., Hamid Q., Kapp A., Leung D.Y., Lipozencic J., Luger T.A., Muraro A., Novak N., Platts-Mills T.A., Rosenwasser L., Scheynius A., Simons F.E., Spergel J., Turjanmaa K., Wahn U., Weidinger S., Werfel T. \& Zuberbier T. 2006. Diagnosis and treatment of atopic dermatitis in children and adults: European Academy of Allergology and Clinical Immunology/ American Academy of Allergy, Asthma and Immunology/PRACTALL Consensus Report. J. Allergy Clin. Immunol. 118(1):152-169.

Burton G., Burrows A., Walker R., Robson D., Bassett R., Bryden S. \& Hill A. 2004. Efficacy of cyclosporin in the treatment of atopic dermatitis in dogs - combined results from two veterinary dermatology referral centres. Aust. Vet. J. 82(11):681-685.

Cork M.J., Robinson D.A., Vasilopoulos Y., Ferguson A., Moustafa M., MacGowan A., Duff G.W., Ward S.J. \& Tazi-Ahnini R. 2006. New perspectives on epidermal barrier dysfunction in atopic dermatitis: Gene-environment interactions. J. Allergy Clin. Immunol. 118(1):3-21.

Deboer D.J. 2004. Canine atopic dermatitis: new targets, new therapies. J. Am. Soc. Nutr. Sci., Madison, 134:8.

Deboer D.J. 2008. Atopic dermatitis: pathogenesis, clinical signs, and diagnosis. North American Veterinary Conference, p.370-371.

Del Rosso J. \& Friedlander S.F. 2005. Corticosteroids: options in the era of steroid-sparing therapy. J. Am. Acad. Dermatolol. 53(1):50-58.

Favrot C. 2009. Clinical signs and diagnosis of canine atopic dermatitis. Eur. J. Companion Anim. Pract. 19:219-222.

Favrot C., Steffan J., Seewald W. \& Picco F. 2010. A prospective study on the clinical features of chronic canine atopic dermatitis and its diagnosis. Vet. Dermatol. 21(1):23-30.

Fontaine J. \& Olivry T. 2001. Treatment of canine atopic dermatitis with cyclosporine: a pilot clinical study. Vet. Rec. 148(21):662-663.

Guaguère E., Steffan J. \& Olivry T. 2004. Cyclosporin A: a new drug in the field of canine dermatology. Vet. Dermatol. 15:61-74.

Hillier A. 2002. Allergy testing and treatment for canine atopic dermatitis. Vet. Med. 97(3):210-224.

Homey B., Steinhoff M., Ruzicka T. \& Leung D.Y. 2006. Cytokines and chemokines orchestrate atopic skin inflammation. J. Allergy Clin. Immunol. 118(1):178-189.

Lucas R., Beviani D., Pelegrini C., Cantagallo K. \& Mingossi R.J. 2007. Avaliação da efetividade do uso da ciclosporina na terapia de cães atópicos. Clínica Veterinária 12(69):68-72.

Nuttal T. 2008. Abordagem da dermatite atópica. Vet. Focus, Dermatologia canina e felina 18:32-39.

Olivry T. \& Mueller R.S. 2003. Evidence-based veterinary dermatology: a systematic review of the pharmacotherapy of canine atopic dermatitis. Vet. Dermatol. 14:121-146.

Olivry T., Rivierre C., Jackson H.A., Murphy K.M., Davidson G. \& Sousa C.A. 2002a. Cyclosporine decreases skin lesions and pruritus in dogs with atopic dermatitis: a blinded randomized prednisolona-controlled trial. Vet. Dermatol. 13(2):77-87.

Olivry T., Steffan J., Fisch R.D., Prélaud P., Guaguère E., Fontaine J. \& Carlotti D.N. 2002b. Randomized controlled trial of the efficacy of cyclosporine in the treatment of atopic dermatitis in dogs. J. Am. Vet. Med. Assoc. 221(3):370-377.

Olivry T., Marsella R., Maeda S., Pucheu-Haston C.M. \& Hammerberg B. 2005. Mechanism of lesion formation in canine atopic dermatitis: 2004 hypothesis, p.10-16. In: Hillier A., Foster A.P. \& Kwochka K.W. (Eds), Advances in Veterinary Dermatology.

Olivry T., Marsella R., Iwasaki T. \& Mueller R. 2007. Task Force on Canine Atopic Dermatitis: evaluation of CADESI-03, a severity scale for clinical trials enrolling dogs with atopic dermatitis. Vet. Dermatol. 18:79-96.

Olivry T., Deboer D.J., Favrot C., Jackson H.A., Mueller R.S., Nuttall T. \& Prélaud P. 2010. Treatment of canine atopic dermatitis: clinical practice guidelines from the International Task Force on Canine Atopic Dermatitis. Vet. Dermatol. 21(3):233-248.

Radowicz S.N. \& Power H.T. 2005. Long-term use of cyclosporine in the treatment of canine atopic dermatitis. Vet. Dermatol. 16:81-86.

Rybnicek J. 2009. Further validation of a pruritus severity scale for use in dogs. Vet. Dermatol. 20(2):115-122.

Steffan J., Strehlau G., Maurer M. \& Rohlfs A. 2004. Cyclosporin A pharmacokinetics and efficacy in the treatment of atopic dermatitis in dogs. J. Vet. Pharmacol. Therapeutics 27(4):231-238.

Steffan J., Parks C. \& Seewald W. 2005. Clinical trial evaluating the efficacy and safety of cyclosporine in dogs with atopic dermatitis. Journal of the Am. Vet. Med. Assoc. 226(11):1855-1863.

Steffan J., Favrot C. \& Mueller R. 2006. A systematic review and meta-analysis of the efficacy and safety of cyclosporin for the treatment of atopic dermatitis in dogs. Vet. Dermatol. 17(1):3-16.

Yazbek A.V.B. \& Larsson C.E. 2012. Avaliação da eficácia da ciclosporina no tratamento de cães atópicos. Braz. J. Vet. Res. Anim. Sci. 49(5):360-366. 